\title{
Hydrophillic Interaction Chromatography-tandem Mass Spectrometry Method for Identification and Quantitation of 5-MeO-DIPT and its Metabolites in Rat Urine
}

\author{
Yoon Kim, ${ }^{\dagger}$ Un Yong Kim, ${ }^{\dagger}$ Moon Kyo In, ${ }^{\dagger}$ Jaeick Lee, ${ }^{\dagger}$ Oh-Seung Kwon,${ }^{\dagger}$ and Hye Hyun Yoo ${ }^{\dagger, s, *}$ \\ ${ }^{\dagger}$ Doping Control Center, Korea Institute of Science and Technology, P.O. Box 131, Cheongryang, Seoul 130-650, Korea \\ 'Drug Analysis Laboratory, Supreme Prosecutors' Office, Seoul, Korea \\ ${ }^{\S}$ College of Pharmacy, Hanyang University, Ansan-si, Gyeonggi-do 426-791, Korea. ${ }^{*}$ E-mail: yoohh@hanyang.ac.kr \\ Received October 2, 2010, Accepted January 29, 2011
}

\begin{abstract}
5-Methoxy- $N, N$-diisopropyltryptamine (5-MeO-DIPT), a psychoactive tryptamine derivative, is a hallucinogenic drug of abuse. In this study, 5-OH-DIPT and its metabolites were identified and the quantitative method was developed and validated by using hydrophilic interaction chromatography-tandem mass spectrometry (HILICMS/MS). Chromatographic separation was achieved on an Atlantis HILIC silica column $(5 \mu \mathrm{m}, 100 \times 2.1 \mathrm{~mm})$. The metabolites of 5-MeO-DIPT in rat urine were characterized via $\mathrm{Q} 1$ scanning and product ion scanning. As a consequence, 5-MeO-IPT, 5-OH-DIPT, 6-OH-5-MeO-DIPT and their glucuronide conjugates were detected and identified as the metabolites of 5-MeO-DIPT. Subsequently, a quantitative method for 5-MeO-DIPT and its major metabolites, 5-MeO-IPT and 5-OH-DIPT, was developed in multiple reactions monitoring (MRM) mode. The calibration curves for all analytes evidenced good linearity over the concentration range of 1$1000 \mathrm{ng} / \mathrm{mL}$ with linear correlation co-efficients $\left(r^{2}\right)$ in excess of 0.99 . The intra- and inter-day accuracy and precision were $92.2-110.2 \%$ and $1.5-9.9 \%$, respectively.
\end{abstract}

Key Words : 5-MeO-DIPT, HILIC-MS/MS, Metabolites, Profiling, Quantitation

\section{Introduction}

Many tryptamine analogues are known to have hallucinogenic properties that exist in plants, fungi, and animals, and can also be synthetically produced. ${ }^{1} 5$-Methoxy- $N, N$-diisopropyltryptamine (5-MeO-DIPT), also referred as "Foxy", is also a tryptamine derivative; Shulgin and Carter were the first to synthesize it and characterize its CNS activity. ${ }^{2}$ 5$\mathrm{MeO}$-DIPT induces pharmacological effects similar to those of other Schedule I hallucinogens such as dimethyltryptamine (DMT) and alpha-ethyltryptamine (AET). ${ }^{3,4}$

There have been several previous reports concerning the metabolism and excretion of 5-MeO-DIPT metabolites in biological samples. According to the relevant literature, ${ }^{5-8} 5-$ MeO-DIPT is primarily metabolized to 5 -hydroxy- $N, N$-diisopropyltryptamine (5-OH-DIPT) and 5-methoxy- $N$-diisopropyltryptamine (5-MeO-IPT) in humans and rats. The chemical structures of the metabolites were characterized in human urine via gas chromatography mass spectrometry $(\mathrm{GC} / \mathrm{MS})^{9,10}$ and liquid chromatography mass spectrometry (LC/MS). ${ }^{5,6,11}$

Based on these previous studies, we recently developed a simultaneous quantitative method for 5-MeO-DIPT, 5-OHDIPT, and 5-MeO-IPT using reverse phase (RP)-LC/MS/ MS. This method employed a conventional $\mathrm{C}_{18}$ column for chromatographic separation, and the retention behavior and peak shape of all analytes was judged sufficient for accurate and reproducible analysis. However, the analytes including 5-MeO-DIPT are relatively hydrophilic, and were eluted at a relatively early stage. Therefore, we considered that the system using a hydrophilic interaction chromatography
(HILIC) is more appropriate for the overall analysis of metabolites, including more polar phase II conjugates than the RP-system. For this reason, we used a hydrophilic interaction (HILIC)-LC/MS/MS system in order to analyze the 5-MeO-DIPT metabolites.

In this study, we profiled the phase I and phase II metabolites of 5-MeO-DIPT found in urine from 5-MeO-DIPTtreated rats using an HILIC-LC/MS/MS system. Additionally, we developed a quantitative method for the detection of 5$\mathrm{MeO}-\mathrm{DIPT}$ and its major metabolites, 5-OH-DIPT and 5MeO-IPT, using an HILIC-LC/MS/MS system.

\section{Experimental}

Chemicals. 5-MeO-DIPT，5-OH-DIPT，5-MeO-IPT，5MeO-DIPT- $d_{4}, 5$-OH-DIPT- $d_{4}$ and 5-MeO-IPT- $d_{4}$ were provided by the Pesticide Chemistry \& Toxicology Laboratory (Seoul National University, Seoul, Korea). Ammonium formate and spectroanalytical-grade acetonitrile were purchased from Fisher J.T. Baker (Phillipsburg, NJ, USA). All other chemicals were of analytical grade, unless otherwise specified. Deionized water was prepared using a Milli-Q-purification system (Millipore; Milford, MA, USA), which was then filtered through a $0.45 \mu \mathrm{M}$ filter for liquid chromatography. High-purity nitrogen (99.999\%) was purchased from Shin Yang Gas Co. (Seoul, Korea).

Animal Experiment and Sample Collection. Male Sprague-Dawley rats weighing approximately $250-280 \mathrm{~g}$ were purchased from the Animal Center of Orient Bio (Kyung-Ki Do, Korea). Rats were maintained at a temperature of $23 \pm 3{ }^{\circ} \mathrm{C}$ and a relative humidity of $50 \pm 10 \%$ with a $12 \mathrm{~h}$ 
light/dark cycle. At least 1 week before being used in the experiments, the rats were permitted to acclimate to the housing conditions. Each rat was individually housed in a rat metabolic cage for 1 day prior to the commencement of the study. 5-MeO-DIPT was dissolved in corn oil and was orally administered to the rats at a dose of $20 \mathrm{mg} / \mathrm{kg}$. Urine samples were collected $6 \mathrm{~h}$ after the administration of the drug. The collected samples were stored at $-20{ }^{\circ} \mathrm{C}$ in a freezer until LC/MS/MS analysis.

Preparation of Calibration and Quality Control Standards. Primary stock solutions of 5-MeO-DIPT, 5-MeO-IPT, and 5OH-DIPT were prepared at $1 \mathrm{mg} / \mathrm{mL}$ in $70 \%$ methanol (v/v). Standard mixture solutions were prepared via serial dilution with $70 \%$ methanol $(\mathrm{v} / \mathrm{v})$ to acquire working standard solutions at the appropriate concentration levels. Calibration and QC standards were prepared by spiking $10 \mu \mathrm{L}$ of the working solution to $990 \mu \mathrm{L}$ of blank rat urine. The concentrations of calibration standards were $1,2,5,10,20,50,100,200$, 500 and $1000 \mathrm{ng} / \mathrm{mL}$, and the QC standards were prepared at concentrations of $2,10,100$, and $1000 \mathrm{ng} / \mathrm{mL}$. The standard samples were stored at $-20{ }^{\circ} \mathrm{C}$ until analysis.

Analytical Method. A $20 \mu \mathrm{L}$ aliquot of rat urine sample was mixed with $10 \mu \mathrm{L}$ of $500 \mathrm{ng} / \mathrm{mL}$ deuterium-labeled compounds (5-MeO-DIPT- $d_{4}, 5-\mathrm{MeO}-\mathrm{IPT}-d_{4}$ and 5-OHDIPT- $d_{4}$ solved in $70 \% \mathrm{MeOH}$ ) as internal standards and $40 \mu \mathrm{L}$ of acetonitrile, then mixed by vortexing for $20 \mathrm{~s}$. The tubes were centrifuged for $6 \mathrm{~min}$ at $10,000 \times g$ and the supernatant $(60 \mu \mathrm{L})$ was transferred into an LC/MS vial. An aliquot $(2 \mu \mathrm{L})$ was injected into the LC/MS/MS system for qualitative or quantitative analysis using an autosampling device.

HILIC-MS/MS. The LC-MS/MS instrument consisted of a Nanospace SI-2 HPLC system (Shiseido, Tokyo, Japan) and an API4000 triple quadrupole mass spectrometer (Applied Biosystems-SCIEX, Concord, Canada). Chromatographic separation was conducted on an Atlantis HILIC silica column $(5 \mu \mathrm{m}, 100 \times 2.1 \mathrm{~mm}$, Waters Co., Milford, MA, USA) which was maintained at $40^{\circ} \mathrm{C}$. The HPLC mobile phases were as follows: A, $10 \mathrm{mM}$ ammonium formate (pH 4) and B, 100\% acetonitrile. Separation was achieved using a gradient flow at a rate of $0.3 \mathrm{~mL} / \mathrm{min}$. The initial composition was $90 \% \mathrm{~B}$, and decreased in a linear fashion to $70 \%$ in $4.5 \mathrm{~min}$, then maintained for $1.0 \mathrm{~min}$, followed by column re-equilibrium for $3.5 \mathrm{~min}$. The total run time was $9.0 \mathrm{~min}$.
The elutes from the column were introduced directly into the turbo ion spray source, which was operated in positive ion mode. Ion spray voltage and nebulizing gas, turbo spray gas, and curtain gas were set at $5500 \mathrm{~V}$ and 50,50 , and 20 psi, respectively. The heated nebulizer temperature was set at $500{ }^{\circ} \mathrm{C}$. Multiple reactions monitoring (MRM) detection was employed with nitrogen used as the collision gas (7 arbitrary values) with a dwell time of $20 \mathrm{~s}$ for each transition. The MRM conditions employed are summarized in Table 1. To acquire the product ion spectra for 5-MeO-DIPT and its metabolites, product ion scans were conducted for all of the postulated metabolite peaks. Data acquisition was conducted using Analyst 1.4.2 software.

Method Validation. The validation method employed included selectivity, linearity, intra- and inter-day accuracy and precision, recovery, and stability. All procedures were conducted in accordance with the "Guidance for industry, Bioanalytical method validation" criteria established by the US Food and Drug Administration (FDA) (2001). ${ }^{12}$

Selectivity. The selectivity of the method was evaluated in blank rat urine. The blank rat urine was checked for the presence of endogenous peaks, which might interfere with the peaks of the analytes. The chromatogram was compared with those of the urine samples spiked with $50 \mathrm{ng} / \mathrm{mL}$ of standards and collected for $0-24 \mathrm{~h}$ after oral administration of 5-MeO-DIPT.

Calibration Curve and Linearity. The calibration curves of 5-MeO-DIPT, 5-OH-DIPT and 5-MeO-IPT in rat urine were generated by plotting the peak area ratio for the analyte to each corresponding IS versus the analyte concentration. The concentration range for all analytes was $1-1000 \mathrm{ng} / \mathrm{mL}$, and the calibration equations were determined via leastsquare linear regression. To assess the linearity, the linear

Table 1. MRM conditions for the analysis of 5-MeO-DIPT and its metabolites

\begin{tabular}{lccc}
\hline \multicolumn{1}{c}{ Analytes } & Q1 mass & Q3 mass & $\begin{array}{c}\text { Collision } \\
\text { energy (V) }\end{array}$ \\
\hline 5-MeO-DIPT & 275 & 174 & 25 \\
5-MeO-IPT (M1) & 233 & 174 & 35 \\
5-OH-DIPT (M2) & 261 & 160 & 32 \\
5-MeO-DIPT- $d_{4}$ (IS) & 279 & 178 & 21 \\
5-MeO-IPT- $d_{4}$ (IS) & 237 & 178 & 22 \\
5-OH-DIPT- $d_{4}$ (IS) & 265 & 164 & 40 \\
\hline
\end{tabular}

Table 2. Chromatographic and mass spectrometric data of 5-MeO-DIPT and its metabolites

\begin{tabular}{|c|c|c|c|}
\hline Analytes & Retention time (min) & {$[\mathrm{M}+\mathrm{H}]^{+}(\mathrm{m} / \mathrm{z})$} & Characteristic ions of $\mathrm{MS}^{2}(\mathrm{~m} / \mathrm{z})$ \\
\hline 5-MeO-DIPT & 4.0 & 275 & $174,114,102$ \\
\hline M1 (5-MeO-IPT) & 4.0 & 233 & $174,162,72$ \\
\hline M2 (5-OH-DIPT) & 4.1 & 261 & $160,114,102$ \\
\hline M3 (6-OH-5-MeO-DIPT) & 4.1 & 291 & $190,114,72$ \\
\hline M4 (5-OH-DIPT-G) & 5.4 & 437 & $336,261,160,114,102$ \\
\hline M5 (6-OH-5-MeO-DIPT-G) & 5.8 & 451 & $392,350,275,174,114$ \\
\hline M6 (5-MeO-DIPT-G) & 4.9 & 467 & 366,114 \\
\hline M7 (5-MeO-DIPT-G) & 5.5 & 467 & $366,291,190,114$ \\
\hline
\end{tabular}


correlation co-efficient $\left(r^{2}\right)$ for the curves and the percent deviation from the nominal concentration were determined.

Precision and Accuracy. The QC samples at 2, 10, 100 and $1000 \mathrm{ng} / \mathrm{mL}$ were assayed in sets of five replicates to evaluate the intra-day precision and accuracy. The procedure was repeated over 5 consecutive days for the inter-day assay. Accuracy was calculated in accordance with the following equation: accuracy $(\%)=$ (measured concentration/ nominal concentration $) \times 100$. The precision was expressed as the percentage co-efficient of variation $(\% \mathrm{CV})$.

Recovery. The recovery was evaluated via analysis of the QC samples (at 10 and $200 \mathrm{ng} / \mathrm{mL}$ ) and comparison of the MS response with the standard mixture solutions at the same concentration. For each run, the samples were analyzed in triplicate.

Stability. The stability of 5-MeO-DIPT, 5-MeO-IPT and 5-OH-DIPT were evaluated using two QC samples (10 and $200 \mathrm{ng} / \mathrm{mL}$ ) in three sets of replicates. For short-term stability, the QC sample was maintained at room temperature for $6 \mathrm{~h}$, and then analyzed. For long-term stability, the QC sample was stored for 14 days at $-70{ }^{\circ} \mathrm{C}$ and subsequently thawed and analyzed. For the freeze/thaw stability test, the QC sample was frozen for $24 \mathrm{~h}$ at $-70{ }^{\circ} \mathrm{C}$ and thawed at room temperature. When completely thawed, this cycle was repeated twice more, after which the sample was analyzed. The post-preparative stability was evaluated by determining the stability of the sample in an autosampler for $24 \mathrm{~h}$.

\section{Results and Discussion}

Qualitative Analysis of 5-MeO-DIPT and its Metabolites in Urine. To characterize the metabolites of 5-MeO-DIPT, the urine samples collected from the rats to which 5-MeODIPT had been orally administered were analyzed via HILIC-MS/MS. The chromatographic and mass spectrometric information for 5-OH-DIPT and its proposed metabolites is summarized in Table 2.

A total of 7 metabolites (M1-M7) were detected in the form of protonated molecular ions via the Q1 scan analysis. These included 5-MeO-IPT (M1), 5-OH-DIPT (M2), and 6OH-5-MeO-DIPT (M3) as phase I metabolites and the glucuronide conjugates of 5-OH-DIPT, 6-OH-5-MeO-DIPT, and 5-MeO-DIPT (M4-M7). Most of the metabolites are more hydrophilic than the parent drug. Therefore, the metabolites were retained longer on the stationary phase than the parent drug; the phase I metabolites (M1-M3) were generally eluted later than the parent compound, and the phase II metabolites (M4-M7) which are more polar than their aglycones followed the phase I metabolites. This elution order in the present HILIC system is opposite to that shown in the RP system.

The sample was subjected to product ion scan analysis in order to elucidate the chemical structures of the metabolites (Figure 1). Among them, M1 (5-MeO-IPT) and M2 (5-OHDIPT) were verified via comparison of the LC-MS/MS data with the reference standards. The $[\mathrm{M}+\mathrm{H}]^{+}$ion of 5-MeODIPT was observed at $\mathrm{m} / \mathrm{z} 275$ and its major product ions were observed at $\mathrm{m} / \mathrm{z} 174, \mathrm{~m} / \mathrm{z} 114$, and $\mathrm{m} / \mathrm{z} 72$. The ion of $\mathrm{m} / \mathrm{z} 174$ was thought to be generated by the loss of a diisopropylamine moiety and the $\mathrm{m} / \mathrm{z} 114$ and $\mathrm{m} / \mathrm{z} 102$ ions are produced by cleavages of the $\mathrm{C}-\mathrm{C}$ and $\mathrm{C}-\mathrm{N}$ bonds of the aliphatic amine group. The $[\mathrm{M}+\mathrm{H}]^{+}$ion of M1 (5-MeO-IPT) was detected at $m / z 233$, and its major product ions were observed at $m / z 174, m / z 162$, and $m / z 72$. The $[\mathrm{M}+\mathrm{H}]^{+}$ion of M2 (5-OH-DIPT) was observed at $\mathrm{m} / z 261$ and its major product ions were observed at $\mathrm{m} / \mathrm{z} 160, \mathrm{~m} / \mathrm{z} 114$, and $\mathrm{m} / \mathrm{z}$ 102. The $[\mathrm{M}+\mathrm{H}]^{+}$ion of $\mathrm{M} 3$ (6-OH-5-MeO-DIPT) was detected at $m / z 291$ and its major product ions were observed at $m / z \quad 190$ and $m / z \quad 114$. Their cleavage patterns of protonated molecular ions in $\mathrm{MS} / \mathrm{MS}$ analysis were all similar to that of 5-MeO-DIPT. M4 was thought to be 5-OHDIPT glucuronide, which showed protonated molecular ions at $m / z 437,176$ Da higher than that observed with 5-OHDIPT. The $[\mathrm{M}+\mathrm{H}]^{+}$ion of M5 was noted at $m / z 451$, which corresponds to 5-MeO-DIPT glucuronide. Two peaks (M6 and M7) were observed at $m / z$ 467, which corresponds to 6$\mathrm{OH}-5-\mathrm{MeO}-\mathrm{DIPT}$ glucuronide. These glucuronide peaks all evidenced fragment patterns similar to their aglycones. Our findings with regard to these metabolites were generally consistent with previous reports. ${ }^{8}$ Among them, 5-MeODIPT glucuronide (M5) and another 6-OH-5-MeO-DIPT glucuronide (M6 or M7) were also reported in this study. However, no sulfate conjugates were detected in our system. This may be attributable to the intrinsic lower sensitivity of sulfate conjugates as compared with glucuronides in ESIpositive mode. $^{5}$

HILIC-MS/MS Method for the Quantitation of 5MeO-DIPT and its Metabolites. Based on the qualitative results generated via HILIC-MS/MS analysis, a quantitative analytical method was developed for 5-MeO-DIPT and its major metabolites, 5-OH-DIPT and 5-MeO-IPT. For all analytes, $[\mathrm{M}+\mathrm{H}]^{+}$ions and those major product ions were selected as precursor and product ion pairs for MRM detection. The deuterium labeled compound for each analyte was employed as an internal standard (IS). The ISs showed MS/MS fragmentations analogous to their corresponding analytes. The representative MRM chromatograms for the blank and standard-spiked urine, and the urine collected from the 5-MeO-DIPT-dosed rats are shown in Figure 2. As compared with our previous study using an RP-LC system, the present method improved the peak shape by reducing the peak tailings observed in the RP-LC-MS/MS chromatogram, and provided a more stable condition for the analysis of polar phase II metabolites. Additionally, in the HILIC system, the efficacy of the ionization at the ESI source and the sensitivity are increased because of its higher organic solvent ratio. ${ }^{13}$ Actually, the HILIC system showed approximately 3 times higher signal to noise $(\mathrm{S} / \mathrm{N})$ ratio at $1 \mathrm{ng} / \mathrm{mL}$, the LLOQ of the current method compared with the RP system with a $\mathrm{C}_{18}$ column.

\section{Validation of LC-MS/MS Analytical Method}

Chromatogram and Selectivity. The specificity of the 

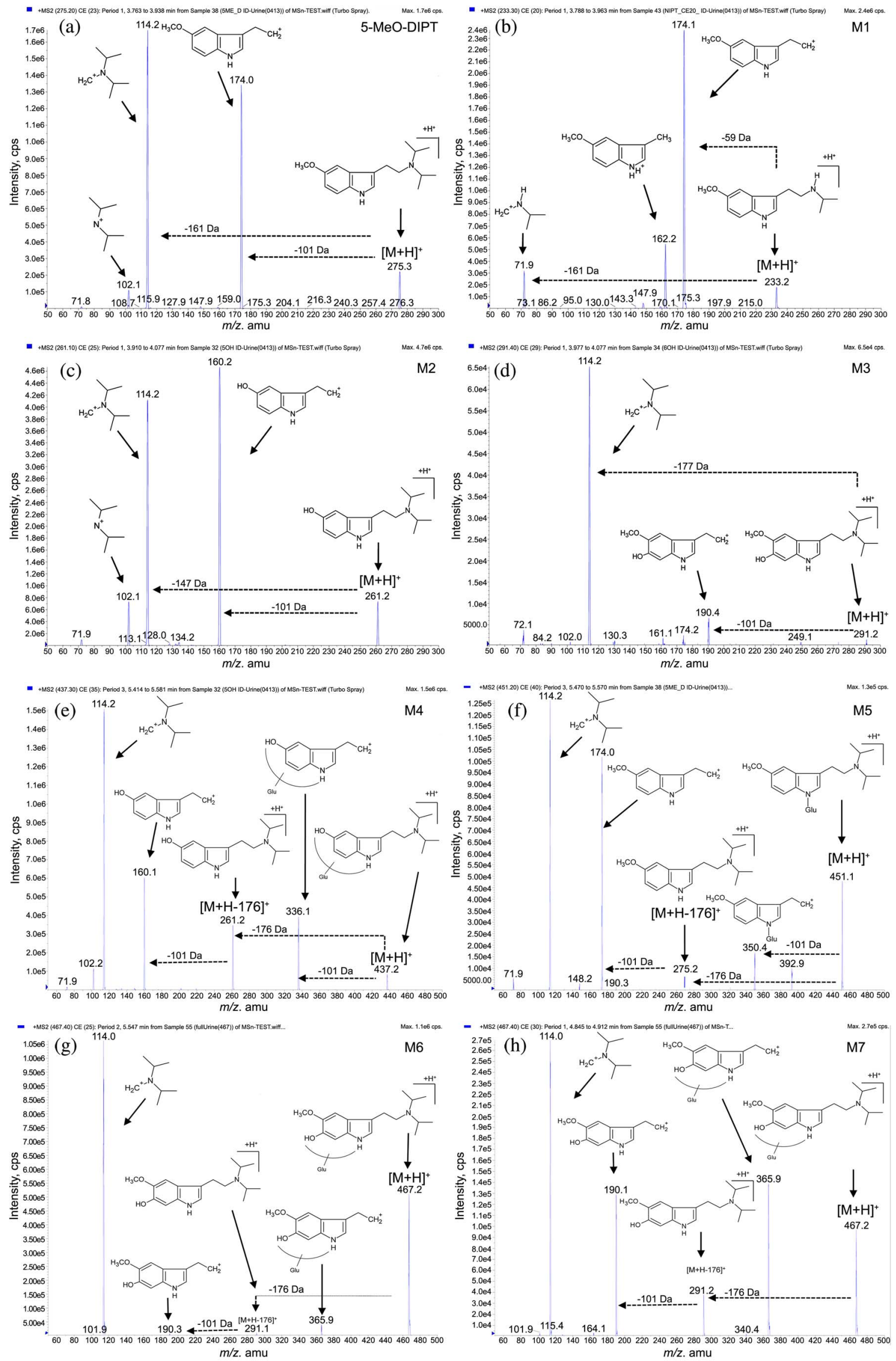

Figure 1. MS/MS spectra and proposed fragmentation mechanism for 5-MeO-DIPT and its metabolites. 


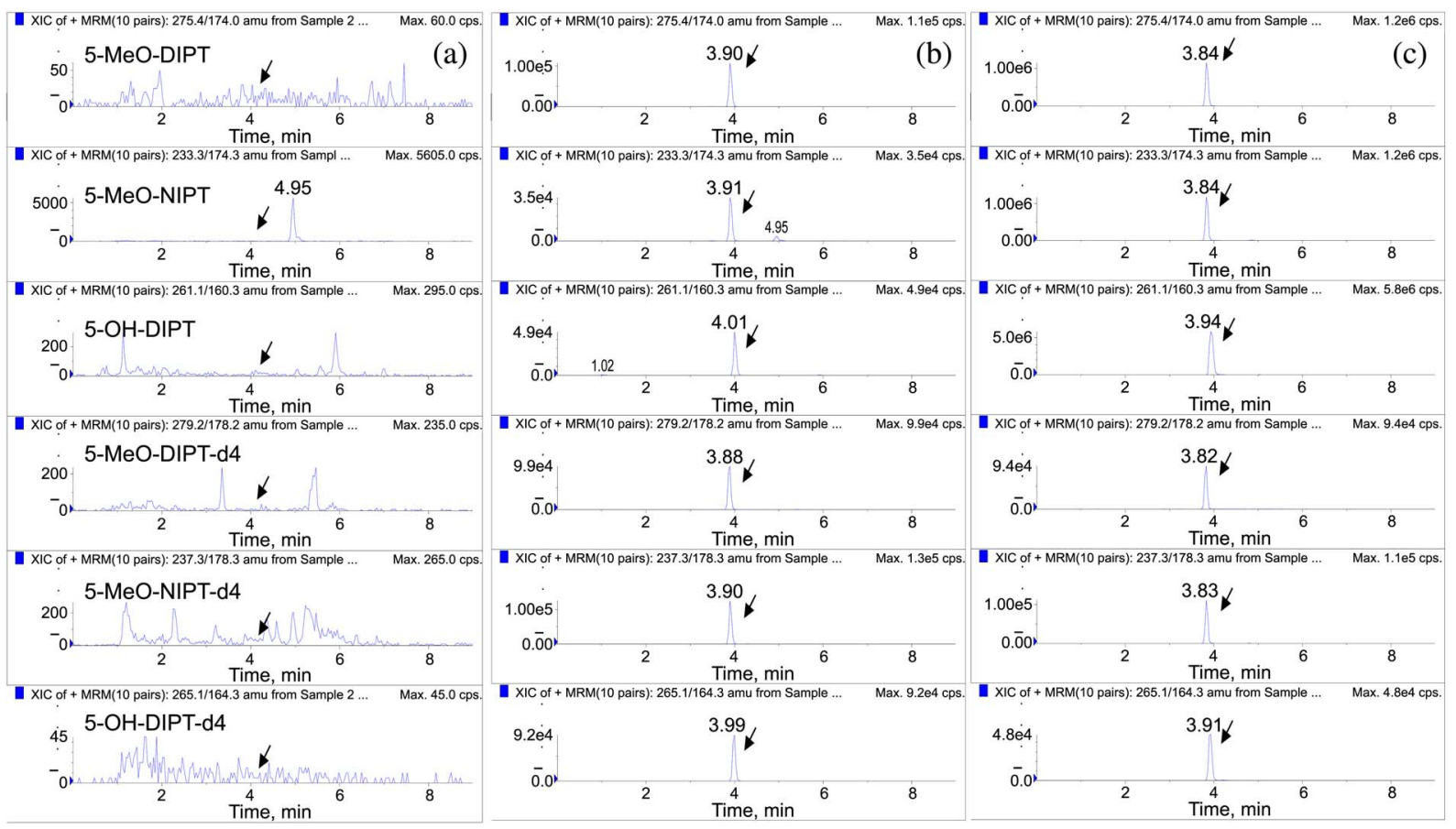

Figure 2. Representative MRM chromatograms of (a) blank urine, (b) urine spiked with standards (50 ng/mL) and deuterium-labeled ISs $(50 \mathrm{ng} / \mathrm{mL})$, and (c) urine collected $24 \mathrm{~h}$ after oral administration of $5-\mathrm{MeO}-\mathrm{DIPT}(20 \mathrm{mg} / \mathrm{kg})$ to rats.

method developed herein was evaluated via comparisons of the MRM chromatograms of the urine samples spiked with the standards and collected from the drug-treated rats with blank urine samples. As shown in Figure 2(a), no interference was detected around any of the analyte peaks in the chromatograms of the blank urine.

Calibration Curve and Linearity. The calibration curves of 5-MeO-DIPT, 5-MeO-IPT, and 5-OH-DIPT were generated by plotting the peak-area ratio of the analyte to IS versus the analyte concentration via least-square regression analysis. The calibration curves for all analytes evidenced good linearity over the concentration range of $1-1000 \mathrm{ng} / \mathrm{mL}$ (Table 3). In our analysis, the lower limit of quantification (LLOQ) was determined as the lowest concentration of the analytes that can be measured with a coefficient of variation and deviation from a nominal value, both less than $20 \%$. The
LLOQs for 5-MeO-DIPT, 5-MeO-IPT and 5-OH-DIPT were all $1.0 \mathrm{ng} / \mathrm{mL}$ (Table 3).

Precision and Accuracy. Intra- and inter- day precision and accuracy were determined via the repeat analysis of QC samples at 2, 10, 100, and $1000 \mathrm{ng} / \mathrm{mL}$. For 5-MeO-DIPT, 5MeO-IPT, and 5-OH-DIPT, the intra-day accuracies ranged between $97.5-110.0 \%, 98.8-110.2 \%$, and $92.2 \%-110.2 \%$, and the coefficients of variation were $2.0-6.8 \%, 2.0-8.2 \%$, and $2.5-4.8 \%$, respectively. The inter-day accuracies fell within ranges of 93.9-97.5\%, 99.3-106.3, and 98.2-101.6\%, and the coefficients of variation were $2.1-6.0 \%, 2.1-9.6 \%$ and $1.5-9.9 \%$, respectively (Table 4). These results satisfied the bioanalytical method validation criteria established by the FDA, ${ }^{12}$ and indicated that the developed method was highly reproducible and precise for the quantitation of 5MeO-DIPT, 5-MeO-IPT and 5-OH-DIPT in urine.

Table 3. Calibration data for 5-MeO-DIPT, 5-MeO-IPT and 5-OH-DIPT in rat urine $(n=3)$

\begin{tabular}{|c|c|c|c|c|c|c|c|c|c|c|c|c|c|c|}
\hline \multirow{2}{*}{ Analytes } & \multirow{2}{*}{ Statistical variable } & \multicolumn{10}{|c|}{ Nominal concentration $(\mathrm{ng} / \mathrm{mL})$} & \multicolumn{3}{|c|}{ Calibration curve } \\
\hline & & 1.0 & 2.0 & 5.0 & 10 & 20 & 50 & 100 & 200 & 500 & 1000 & Slope $\mathrm{A}^{a}$ & Intercept $\mathrm{B}^{b}$ & $r^{2}$ \\
\hline \multirow{3}{*}{ 5-MeO-DIPT } & Mean (ng/mL) & 1.0 & 1.9 & 5.1 & 10.7 & 20.5 & 52.0 & 99.2 & 200.7 & 494.3 & 1000.3 & 0.00498 & 0.00234 & 0.9997 \\
\hline & Accuracy (\%) & 98.7 & 93.0 & 101.6 & 107.3 & 102.7 & 104.1 & 99.2 & 100.3 & 98.9 & 100.0 & & & \\
\hline & CV $(\%)$ & 4.9 & 9.8 & 7.5 & 6.6 & 12.8 & 11.6 & 7.7 & 7.2 & 1.3 & 1.7 & & & \\
\hline \multirow{3}{*}{ 5-MeO-IPT } & Mean (ng/mL) & 1.0 & 2.0 & 4.9 & 10.7 & 21.3 & 53.0 & 102.0 & 201.7 & 484.0 & 1008.0 & 0.00149 & 0.00071 & 0.9995 \\
\hline & Accuracy (\%) & 103.9 & 101.3 & 97.3 & 106.7 & 106.7 & 106.1 & 102.0 & 100.8 & 96.8 & 100.8 & & & \\
\hline & CV (\%) & 12.7 & 5.4 & 9.3 & 5.5 & 2.2 & 1.8 & 1.0 & 2.7 & 4.2 & 2.3 & & & \\
\hline \multirow{3}{*}{ 5-OH-DIPT } & Mean (ng/mL) & 1.0 & 2.0 & 5.2 & 10.5 & 21.6 & 53.2 & 103.0 & 195.7 & 491.0 & 1005.7 & 0.00229 & 0.00118 & 0.9999 \\
\hline & Accuracy (\%) & 104.3 & 97.8 & 103.1 & 105.3 & 107.8 & 106.4 & 103.0 & 97.8 & 98.2 & 100.6 & & & \\
\hline & $\mathrm{CV}(\%)$ & 0.6 & 13.5 & 5.9 & 2.7 & 1.9 & 1.4 & 3.5 & 4.3 & 1.1 & 0.7 & & & \\
\hline
\end{tabular}

${ }^{a}$ Values are mean of three calibration curves. ${ }^{b}$ Slope and intercept refer to the regression equation, $y=\mathrm{A} x+\mathrm{B}$ 
Table 4. Intra- and inter-day accuracies and precisions of 5-MeO-DIPT, 5-MeO-IPT and 5-OH-DIPT

\begin{tabular}{|c|c|c|c|c|c|c|c|}
\hline \multirow[b]{2}{*}{ Analytes } & \multirow{2}{*}{$\begin{array}{c}\text { Nominal } \\
\text { concentration } \\
(\mathrm{ng} / \mathrm{mL})\end{array}$} & \multicolumn{3}{|c|}{ Intra-day $(n=5)$} & \multicolumn{3}{|c|}{ Inter-day $(n=5)$} \\
\hline & & $\begin{array}{c}\text { Concentration } \\
\text { found }^{a}(\mathrm{ng} / \mathrm{mL})\end{array}$ & Accuracy (\%) & CV (\%) & $\begin{array}{c}\text { Concentration } \\
\text { found }^{a}(\mathrm{ng} / \mathrm{mL})\end{array}$ & Accuracy $(\%)$ & CV (\%) \\
\hline \multirow{4}{*}{ 5-MeO-DIPT } & 2 & $2.0 \pm 0.1$ & 97.5 & 6.8 & $2.0 \pm 0.1$ & 97.5 & 3.6 \\
\hline & 10 & $10.9 \pm 0.3$ & 109.4 & 2.5 & $9.7 \pm 0.6$ & 97.0 & 6.0 \\
\hline & 100 & $107.5 \pm 5.6$ & 107.5 & 5.2 & $95.2 \pm 2.5$ & 95.2 & 2.6 \\
\hline & 1000 & $1100.0 \pm 22.4$ & 110.0 & 2.0 & $939.3 \pm 20.1$ & 93.9 & 2.1 \\
\hline \multirow{4}{*}{ 5-MeO-IPT } & 2 & $2.0 \pm 0.1$ & 98.8 & 4.5 & $2.0 \pm 0.2$ & 99.3 & 9.6 \\
\hline & 10 & $11.0 \pm 0.3$ & 110.2 & 2.7 & $10.6 \pm 0.2$ & 106.3 & 2.1 \\
\hline & 100 & $104.4 \pm 8.6$ & 104.4 & 8.2 & $104.3 \pm 5.0$ & 104.3 & 4.8 \\
\hline & 1000 & $1092.0 \pm 21.7$ & 109.2 & 2.0 & $1029.8 \pm 47.5$ & 103.0 & 4.6 \\
\hline \multirow{4}{*}{ 5-OH-DIPT } & 2 & $1.8 \pm 0.1$ & 92.2 & 4.8 & $2.0 \pm 0.1$ & 99.1 & 5.0 \\
\hline & 10 & $10.8 \pm 0.4$ & 107.6 & 3.6 & $10.2 \pm 1.0$ & 101.6 & 9.9 \\
\hline & 100 & $105.8 \pm 4.5$ & 105.8 & 4.3 & $98.2 \pm 1.5$ & 98.2 & 1.5 \\
\hline & 1000 & $1102.0 \pm 27.7$ & 110.2 & 2.5 & $984.8 \pm 19.4$ & 98.5 & 2.0 \\
\hline
\end{tabular}

Recovery. The extraction recovery of 5-MeO-DIPT, 5$\mathrm{MeO}-\mathrm{IPT}$, and 5-OH-DIPT from rat urine was more than $88 \%$ at the two QC levels (10 and $200 \mathrm{ng} / \mathrm{mL}$ ) (Table 5). Therefore, this method was regarded as effective for the analysis of analytes in urine. Furthermore, such high recovery values reflect the absence of any significant matrix effect for all analytes.

Stability. The stability of 5-MeO-DIPT, 5-MeO-IPT, and 5-OH-DIPT were evaluated under a variety of process and preservation conditions. The results of all stability tests are shown in Table 6. 5-MeO-DIPT, 5-MeO-IPT, and 5-OHDIPT evidenced good stability $(<90 \%)$ throughout all stages of the analysis. Therefore, the method developed herein was considered applicable for routine analysis.
Application to the Urine Sample from 5-MeO-DIPT Treated Rats. The urine sample collected from rats after oral administration of 5-MeO-DIPT was analyzed using the developed method. 5-MeO-DIPT, 5-MeO-IPT, and 5-OHDIPT were all detected and their concentrations were 6.2 , 7.1 , and $18.8 \mu \mathrm{g} / \mathrm{mL}$, respectively. These results were com-

Table 5. Recoveries of 5-MeO-DIPT, 5-MeO-IPT and 5-OH-DIPT in rat urine $(n=3)$

\begin{tabular}{cccc}
\hline \multirow{2}{*}{$\begin{array}{c}\text { Nominal conc. } \\
(\mathrm{ng} / \mathrm{mL})\end{array}$} & \multicolumn{3}{c}{ Recovery $(\%)$} \\
\cline { 2 - 4 } & 5 -MeO-DIPT & 5-MeO-IPT & 5-OH-DIPT \\
\hline 10 & $92.7 \pm 1.2$ & $100.0 \pm 4.7$ & $88.4 \pm 2.5$ \\
200 & $100.0 \pm 4.0$ & $101.8 \pm 3.7$ & $100.0 \pm 4.0$ \\
\hline
\end{tabular}

Table 6. Stability of 5-MeO-DIPT, 5-MeO-IPT and 5-OH-DIPT in rat urine $(n=3)$

\begin{tabular}{|c|c|c|c|c|c|c|}
\hline \multirow{3}{*}{$\begin{array}{l}\text { Statistical } \\
\text { variable }\end{array}$} & \multicolumn{6}{|c|}{ Nominal conc. $(\mathrm{ng} / \mathrm{mL})$} \\
\hline & \multicolumn{2}{|c|}{ 5-MeO-DIPT } & \multicolumn{2}{|c|}{ 5-MeO-IPT } & \multicolumn{2}{|c|}{ 5-OH-DIPT } \\
\hline & 10 & 200 & 10 & 200 & 10 & 200 \\
\hline \multicolumn{7}{|c|}{ Freeze and thaw stability } \\
\hline Mean (ng/mL) & 11.1 & 223.1 & 9.9 & 205.6 & 10.5 & 223.9 \\
\hline Accuracy $(\%)$ & 111.4 & 111.5 & 99.2 & 102.8 & 105.4 & 112.0 \\
\hline CV $(\%)$ & 2.0 & 1.9 & 10.6 & 3.4 & 2.4 & 1.2 \\
\hline \multicolumn{7}{|c|}{ Short-term stability temperature stability (6 h, room temperature) } \\
\hline Mean (ng/mL) & 10.0 & 214.4 & 9.9 & 195.5 & 10.2 & 211.4 \\
\hline Accuracy $(\%)$ & 99.9 & 107.2 & 98.6 & 97.7 & 102.3 & 105.7 \\
\hline $\mathrm{CV}(\%)$ & 3.5 & 2.5 & 10.6 & 4.5 & 4.7 & 3.4 \\
\hline \multicolumn{7}{|c|}{ Long-term temperature stability $\left(14\right.$ days, $\left.-70^{\circ} \mathrm{C}\right)$} \\
\hline Mean (ng/mL) & 9.2 & 194.2 & 9.9 & 225.2 & 10.5 & 219.7 \\
\hline Accuracy $(\%)$ & 92.0 & 97.1 & 98.6 & 112.6 & 104.7 & 109.9 \\
\hline $\mathrm{CV}(\%)$ & 1.9 & 3.7 & 6.2 & 1.4 & 5.4 & 1.1 \\
\hline \multicolumn{7}{|c|}{ Post-preparative stability ( $24 \mathrm{~h}$, autosampler) } \\
\hline Mean (ng/mL) & 10.1 & 206.3 & 10.0 & 203.3 & 10.7 & 206.3 \\
\hline Accuracy (\%) & 100.8 & 103.2 & 100.0 & 101.7 & 107.3 & 103.2 \\
\hline CV (\%) & 2.8 & 3.4 & 4.7 & 1.0 & 2.8 & 3.4 \\
\hline
\end{tabular}


parable to those obtained from our previous study using a conventional RP-LC-MS/MS system.

\section{Conclusions}

The metabolites of 5-MeO-DIPT in rat urine were characterized using a HILIC-MS/MS system. In this study, 5-MeO-DIPT glucuronide and another 6-OH-5-MeO-DIPT glucuronide were additionally detected aside from the previously reported metabolites. In addition, the quantitative method for the analysis of 5-MeO-DIPT and its major metabolites, 5-MeO-IPT and 5-OH-DIPT, were developed and successfully validated using the HILIC-MS/MS system. The present method improved the peak shape and provided more stable conditions for the analysis of polar phase II metabolites, as compared with RP-LC-MS analysis. Taken together, our results show that this HILIC-MS/MS method should prove useful in the detection and confirmation of 5MeO-DIPT and its metabolites in biological samples.

Acknowledgments. This work was supported in part by grants from the Korea Institute of Science and Technology and in part by a grant (M10640010000-06N4001-00100) from the National R\&D Program of the Ministry of Education, Science, and Technology (MEST) and the National Research Foundation (NRF) of Korea.

\section{References}

1. Kikura-Hanajiri, R.; Hayashi, M.; Saisho, K.; Goda, Y. J. Chromatogra. B 2005, 825, 29.

2. Shulgin, A. T.; Carter, M. F. Commun. Psychopharmacol. 1980, 4, 363.

3. Fantegrossi, W. E.; Harrington, A. W.; Kiessel, C. L.; Eckler, J. R.; Rabin, R. A.; Winter, J. C.; Coop, A.; Rice, K. C.; Woods, J. H. Pharmacol. Biochem. Behav. 2006, 83, 122.

4. Leonhart, M. M. Fed. Regist. 2004, 69, 58050.

5. Kamata, T.; Katagi, M.; Kamata, H. T.; Miki, A.; Shima, N.; Zaitsu, K.; Nishikawa, M. L; Tanaka, E.; Honda, K.; Tsuchihashi, H. Drug. Metab. Dispos. 2006, 34, 512.

6. Narimatsu, S.; Yonemoto, R.; Saito, K.; Takaya, K.; Kumamoto, T.; Ishikawa, T.; Asanuma, M.; Funada, M.; Kiryu, K.; Naito, S.; Yoshida, Y.; Yamamoto, S.; Hanioka, N. Biochem. Pharmacol. 2006, 71, 1377.

7. Wilson, J. M.; McGeorge, F.; Smolinske, S.; Meatherall, R. Forensic. Sci. Int. 2005, 148, 31.

8. Tanaka, E.; Kamata, T.; Ktagi, M.; Tsuchihashi, H.; Honda, K. Forensi. Sci. Int. 2006, 163, 152.

9. Knanmori, T.; Kuwayama, K.; Tsujikawa, K.; Miyaguchi, H.; Iwata, Y.; Inoue, H.; Kishi, T. J. Health Sci. 2006, 52, 425.

10. Vorce, S. P.; Sklerov, J. H. J. Anal. Toxicol. 2004, 28, 407.

11. Chen, B. H.; Liu, J. T.; Chen, W. X.; Chen, H. M.; Lin, C. H. Talanta. 2008, 74, 512.

12. http://www.fda.gov/downloads/Drugs/.../UCM070107.pdf

13. Richard, B. C. Electrospray and MALDI Mass Spectrometry, 2nd ed.; Wiley: MA, U.S.A., 2010; pp 13-19. 